\title{
LE MUSÉE DÉPARTEMENTAL ARLES ANTIQUE ET SON EXTENSION
}

\author{
CLAUDE SINTÈS \\ claude.sintes@,cg13.fr \\ MUSÉE DÉPARTEMENTAL ARLES ANTIQUE \\ ALAIN CHARRON \\ alain.charron@.cg13.fr \\ MUSÉE DÉPARTEMENTAL ARLES ANTIQUE \\ SABRINA MARLIER \\ sabrina.marlier@,cg13.fr \\ MusÉE DÉPARTEMENTAL ARLES ANTIQUe - CD13 CENTRE CAMILle Jullian, \\ CNRS \\ DAVID DJAOUI \\ david.djaoui@.cg13.fr \\ MuSÉE DÉPARTEMENTAL ARLES ANTIQUE - CD13 CENTRE CAMILle JULlian, \\ CNRS \\ http://dx.doi.org/10.25267/Riparia_sup.2018.11.14
}

\section{Le musée départemental Arles antique et les fouilles du Rhône}

L'histoire urbaine d'Arles est assez bien connue en raison des monuments célèbres que la ville abrite et des nombreuses fouilles terrestres qui y sont conduites. Le musée départemental Arles antique, ouvert en 1995, présente une bonne part de ces découvertes, rassemblant ainsi une des plus belles collections archéologiques du pays. Mais si les sarcophages, les mosaïques ou les fragments architecturés y sont nombreux, la vie économique et la puissance du port fluviomaritime n'étaient en revanche qu'évoqués. Des infrastructures étendues, une place commerciale de premier plan redistribuant les richesses des provinces de l'Empire, des chantiers navals efficaces, des corporations d'armateurs ou de bateliers, tout cela ne pouvait qu'être entrevu à travers les textes et les inscriptions dont Arles est riche, sans pouvoir disposer d'un mobilier archéologique suffisant pour préciser les datations ou les provenances.

Depuis 25 ans, les choses ont bien changé : le Rhône est désormais le terrain de fouille le plus productif de la cité et les avancées historiques, que les milliers d'objets remontés autorisent, ont renouvelé radicalement la vision que l'on pouvait avoir. Sous l'égide du Drassm (Département des recherches en archéologie subaquatique et sous marine / ministère de la Culture et de la Communication), associé au musée départemental Arles antique, les directeurs de fouilles Luc Long, Sabrina Marlier, Sandra Greck, David Djaoui et Mourad El Amouri donnent jour après jour une image convaincante des échanges hauturiers ou locaux, des trafics économiques et des outils utilisés.

La moisson est belle car le Rhône, si décrié pour ses crues ravageuses et sa pollution, est un merveilleux protecteur du patrimoine. Ses eaux douces n'abritent pas les espèces dévoreuses de bois ou de marbre que l'on rencontre en mer, son courant uniforme ne maltraite pas les sculptures au point d'arrondir et de polir les pierres les plus dures, son manque de visibilité (quelques dizaines de centimètres la plupart du temps) et ses dangers empêchent le pillage généralisé que les eaux claires de la Méditerranée connaissent depuis l'invention du scaphandre autonome. Si des centaines de milliers d'objets gisent aujourd'hui au fond du fleuve, c'est aussi en raison de l'espace restreint que constitue le port fluvial, de ses sept cent ans d'usage 
et de ses échanges commerciaux intenses. La moisson est belle, disait-on, qu'on en juge : une unique fenêtre de moins de 200 mètres carrés ouverte dans le limon en 2011, à l'occasion du chantier de fouille-relevage de l'épave Arles-Rhône 3, a livré près de 3000 objets répertoriés au musée, 1200 caisses de matériel conservé dans le dépôt de fouille et 120 tonnes de tessons divers, qu'il a fallu remettre dans le fleuve, après comptage et analyses, à l'emplacement même de l'épave, une fois celle-ci prélevée (Marlier (dir.) 2014).

Un ensemble aussi exceptionnel de données se devait d'être exploité au mieux afin que la communauté scientifique en bénéficie et l'intègre à son tour dans des études générales. Les équipes de chercheurs du musée et du Drassm, associés à l'Université et au CNRS, au premier rang duquel le laboratoire du Centre Camille Julian, sont donc engagées dans un processus d'inventaire, d'analyses et de publications de premier ordre. Réserver cette matière aux seuls spécialistes eut été cependant dommageable en raison des budgets publics importants affectés à cette recherche. Le Conseil départemental des Bouches du Rhône, conscient de ses obligations, s'est donc doté de moyens conséquents afin de proposer aux visiteurs une synthèse précise mais assimilable de ces nouvelles connaissances.

Une première exposition «César, le Rhône pour mémoire » a offert en 2009-2010, le bilan d'une vingtaine d'années d'explorations (Long, Picard (dir.) 2009). Sans renoncer un seul instant à la rigueur scientifique nécessaire, la présentation d'objets spectaculaires (le portrait présumé de César en étant le plus emblématique), une mise en espace soigneusement scénographiée mais aussi l'évocation de ce que les journalistes se plaisent à nommer "l'aventure archéologique », a touché le public audelà de toute prévision: près de 400000 personnes se sont passionnées pour ce dossier, montrant à tous, s'il était nécessaire, que l'archéologie est désormais un enjeu aux répercutions sociétales ou économiques, bien loin du seul cabinet feutré des savants.

Cette prise de conscience, renforcée par l'attribution du titre de Capitale européenne de la culture à Marseille-Provence pour l'année 2013, a permis d'envisager la présentation pérenne d'une partie des collections déjà découvertes mais aussi de rendre accessible le projet fou d'une exhumation et d'une restauration d'une épave repérée dans le fleuve depuis plusieurs années. En effet, lorsqu'en 2004 un flanc d'épave est apparu dans les eaux glauques du Rhône, bien peu de gens pouvaient imaginer qu'un chaland long de 31 mètres, quasiment entier, se dissimulait sous les limons du fleuve (Djaoui, Greck, Marlier (dir.) 2011). Et bien moins encore pouvaient croire que ce vestige unique trouverait sa place dans le musée départemental Arles antique agrandi pour l'occasion, quelques années plus tard.

Avec cette opération en tout point exceptionnelle, la barge gallo-romaine ArlesRhône 3, nom de code donné par les archéologues, allait rejoindre le club très fermé des bateaux trouvés complet (ou presque) en fouille, sauvés et installés dans un musée. S'il existe d'assez nombreux fonds de carènes ou fragments de navires présentés dans le monde, seuls les navires royaux V asa de Stockholm et Mary Rose de Portsmouth, la jonque Nanhai 1 de Canton, les bateaux vikings de Roskilde et le chaland Arles-Rhône 3 répondent à cette définition.

La décision politique de l'opération a été prise en octobre 2010, avec pour objectif une inauguration en octobre 2013. Au vu de cette contrainte de temps, c'est un pari insensé qui a été tenté et, contre toute attente, réussi : sortir de l'eau, en moins de sept mois, 11 tonnes d'un bois fragile comme du verre sans en briser la moindre partie ; restaurer l'ensemble (par l'atelier ARC-Nucléart et la société A-Corros) dans 
des délais - moins de deux ans - défiant toutes les normes; installer ce chaland, mais aussi plus de 450 objets permettant d'en comprendre le contexte, dans une aile de $800 \mathrm{~m}^{2}$ spécialement construite pour l'occasion. Et tout cela en moins de trois ans alors que des opérations du même type, pour les bateaux cités précédemment, avaient duré plusieurs décennies. Et tout cela alors que les archéologues n'avaient pas idée des techniques de relevage qu'il leur faudrait employer, alors que les restaurateurs n'avaient jamais traité autant de bois d'un coup, alors que les architectes devaient élaborer leurs plans sans connaitre les dimensions exactes du chaland encore enfoui!

Aujourd'hui, la nouvelle aile du musée de l'Arles antique présente, autour du chaland Arles-Rhône 3, un parcours dédié à la navigation, au commerce et aux activités du port fluviomaritime d'Arles de l'époque romaine. Exceptionnels, voire uniques comme de nombreux objets en bois, ils sont des témoignages inestimables de l'activité commerciale de la cité durant l'époque romaine.

\section{L'aile dédiée au port fluviomaritime d'Arelate}

\section{Le chaland Arles-Rhône 3}

Longue de $31 \mathrm{~m}$ et large d'un peu moins de $3 \mathrm{~m}$, cette épave est celle d'un chaland gallo-romain quasiment entièrement conservé et contenant encore sa dernière cargaison, son mobilier de bord, ses équipements de navigation (un mât de halage, une pelle de gouverne et des perches) et même sa monnaie votive (un denier en argent).

Ce chaland, possiblement construit dans les chantiers navals d'Arles au milieu du I ${ }^{\text {er }}$ siècle, assurait des transports de cargaison dans la section inférieure du Rhône. Lors de son dernier voyage, il descendait le fleuve avec, à son bord, une cargaison de pierres de construction provenant des carrières de Saint-Gabriel, situées près de Tarascon, à une quinzaine de kilomètres au nord d'Arles. Arrivé là, le chaland a fait une halte dans le port, soit pour y décharger ses pierres, soit pour se ravitailler et y passer la nuit avant de reprendre le fleuve. Mais, probablement amarré trop court, et alourdi par ses 21 tonnes de pierres calcaires, le chaland a été englouti dans les eaux du Rhône, sans doute au cours d'un épisode de crue. Les sédiments du fleuve l'ont ainsi recouvert et conservé intact durant près de 2000 ans...

Cette épave, aujourd'hui classée trésor national par le ministère de la Culture, représente ainsi un témoignage exceptionnel du point de la vue de la batellerie galloromaine et de l'économie des transports dans un espace circonscrit à Arles et son territoire.

\section{La navigation}

La section consacrée aux bateaux rend compte de la navigation sur le fleuve. A l'époque romaine, Arles était un port de rupture de charge important: les barges fluviales et les bateaux fluviomaritimes apportaient vers la Méditerranée les productions de Gaule et remontaient vers le nord les marchandises venues de tout l'Empire. La maquette d'un navire à grandes jarres, des dolia, permet de présenter au public un type de bateau fluviomaritime.

Divers équipements de navigation viennent compléter l'exposition. On compte ainsi deux ancres composées de bois, fer et plomb, d'autres sont en pierre et en fer. Quelques éléments d'accastillage proviennent des bateaux qui mouillaient dans le port d'Arles ou en mer, au niveau des Saintes-Maries-de-la-Mer : chaumards à tête de canard, plomb de sonde ainsi qu'une exceptionnelle poulie de bois à âme de bronze. 
Une seconde maquette présente l'épave Arles-Rhône 3 en cours de fouille, avant son relevage. Quelques éléments du chaland, trop fragiles ou trop petits pour être replacés sur le bateau, ont pris place dans une vitrine : quelques-uns des mille sept cents clous en fer, un chiffon de laine poissé ayant servi à l'étanchéité de la coque et, surtout, la monnaie votive du bateau.

\section{Le commerce}

Le commerce fluviomaritime rend compte du rôle essentiel joué par la cité, carrefour de voies romaines et première étape pour les bateaux remontant le Rhône.

Les amphores sont les récipients les plus communément transportés sur le fleuve; l'institution arlésienne en conserve une collection exceptionnelle. Elles sont exposées chronologiquement, depuis le Haut Empire jusqu'à l'Antiquité tardive, le long du chaland, ou présentées en fonction de leurs lieux de production. Sur les cols de certaines, des inscriptions peintes indiquent la qualité des produits ou encore les noms des négociants. Des vitrines abritent des bouchons en liège encore scellés au col de l'amphore.

Cinq vitrines permettent de découvrir les principaux vases de transport et la vaisselle de table provenant de Gaule, d'Italie, d'Espagne, d'Afrique du nord et de Méditerranée orientale.

La Gaule se distingue par des sigillées sud-gauloises de l'Aveyron, avec notamment de grandes coupes au décor moulé provenant d'un même décorateur, un certain Germanus, et un gobelet en sigillée Claire B de la vallée du Rhône avec un médaillon d'applique illustrant l'Apothéose d'Hercule. L'Italie est représentée par plusieurs vases précieux en bronze provenant de Campanie, notamment des pichets, une casserole et une grande amphore de bronze dont les anses sont décorées de chiens de mer. Parmi les céramiques figurent de nombreuses lampes à huile de très belle facture et de nombreuses petites conserves de poisson appartenant aux marins : sard(inae) peut-on lire sur l'un des pots. Des verres exceptionnels d'époque augustéenne, une assiette mosaïquée et une coupe imitant l'onyx résultent de fouilles terrestres réalisées à Cadillan, près de Graveson. L'Espagne offre une superbe collection de parois fines en présentant plus d'une vingtaine de gobelets. Retrouvés miraculeusement encore empilés les uns sur les autres dans les profondeurs du Rhône, ils sont présentés comme tels. L'Afrique du Nord rassemble un service complet avec de grandes et hautes marmites, une partie de sa céramique fine, des cruches et des lampes à huile La Méditerranée orientale est bien représentée avec notamment des amphores à fruit de Gaza, de Mer Noire, de Crète et des objets plus étonnants comme un vase plastique avec une tête de Bacchus ou encore une lampe anthropomorphe à tête et pattes de chat.

Un ensemble de matières premières est aussi présenté. De nombreuses pièces en bronze ont été remontées par des pêcheurs des Saintes-Maries-de-la-Mer et durant les campagnes de carte archéologique conduites par le Drassm. Cette concentration de découvertes circonscrite dans un même secteur pourrait attester de la présence de la cargaison secondaire d'une épave qui reste à découvrir. Des objets de grande qualité en proviennent, notamment une série d'anses, divers vases ou encore un couvercle à poignée en forme de dauphin. D'autres éléments en bronze provenant du Rhône, bras de statue, éléments de toge dorée, résultent de sculptures monumentales Les minerais sont représentés par les cargaisons de nombreuses épaves qui se sont échouées à l'embouchure d'un ancien bras du Rhône : lingots de cuivre, d'étain, de plomb et des barres de fer rassemblent des échantillons de plus d'une trentaine d'épaves. Des pierres d'Urnaginum (situé au nord d'Arles) provenant du chaland 
Arles-Rhône 3 et un sarcophage inachevé de Beaucaire, transporté également par bateau, montrent l'importance des carrières locales.

\section{Le port antique et ses métiers}

La section consacrée au port est dominée par la statue de Neptune. La sculpture du dieu fut choisie pour être dédiée à une corporation de bateliers, les lenunclarii, ce qu'atteste une dédicace inscrite sur le socle.

Cette section permet d'évoquer les métiers liés au port et à ses infrastructures : les chantiers navals, les entrepôts, les ateliers, les bureaux pour l'administration, etc.

Outre les lenunclarii, une autre corporation, les utriculaires, liée au transport de marchandises au moyen d'outres, est évoquée par le sarcophage de l'un de ses membres. La stèle honorifique d'Agricola, procurateur des Augustes pour le service de l'annone (le ravitaillement de Rome en céréales) en Narbonnaise à la fin du $\mathrm{II}^{\mathrm{e}}$ siècle, distingue le travail des armateurs pour des transports maritimes sur de grandes distances, les naviculaires, en mentionnant un de leurs patrons. Enfin, l'esquisse d'un bateau sur la stèle funéraire d'Hermia laisse penser que ce personnage exerçait une activité sur le fleuve.

Sur les quais s'activaient dockers et manutentionnaires. Un relief montre des emballeurs ficelant un ballot et deux terres cuites présentent un personnage portant une outre et un second serrant une amphore entre ses jambes. Des plaquettes en bois, qui servaient peut-être à comptabiliser les chargements, accompagnent ces figurines. Les petits métiers du port sont illustrés par une hache, des semelles et de nombreuses alènes qui servaient à raccommoder voiles et filets.

Deux seaux complètent la présentation. Le premier, provenant du Rhône, est constitué d'un cordage enroulé et d'une poignée en bois. Couvert de poix, il est très certainement le témoignage d'un ouvrier calfateur, tout comme le badigeon de chiffon contenant des cailloux ayant servi à étaler la poix sur les coques des bateaux. Le second, en bois, découvert aux Saintes-Maries-de-la-Mer, est rempli de clous en fer.

Le musée a la chance de conserver l'épitaphe d'un "douanier », Apronianus, chargé de percevoir le " $40^{\mathrm{e}}$ des Gaules", un impôt levé sur toutes les marchandises importées. Des poids et balances, instruments de mesures contrôlés par l'administration, servaient à vérifier les échanges. Les services administratifs sont également évoqués par des plombs qui scellaient les marchandises. Certains donnent des indications sur la provenance des chargements : la Palestine, Djerba ou encore l'Anatolie.

L'activité commerciale sur le fleuve a perduré tout au long de l'Histoire et les visiteurs peuvent découvrir, grâce à la longue ouverture pratiquée dans la façade de l'extension, des péniches et des bateaux fluviomaritimes allant vers le port actuel de la ville, installé plus au nord. Deux mille ans plus tard, Arles est toujours un port et le Rhône continue à jouer un rôle essentiel dans la vie de la cité.

\section{Bibliographie}

D. Djaoui, S. Greck, S. MARLier (dir.), Arles-Rhône 3. Le naufrage d'un chaland antique dans le Rhône, enquête pluridisciplinaire, Arles, Musée départemental Arles antique, Actes Sud, 2011. 
S. MARLIER S. (dir.), Arles-Rhône 3, un chaland gallo-romain du I $I^{\mathrm{er}}$ siècle apr. Jésus-Christ, Paris, CNRS Editions, Musée départemental Arles antique, 2014 (Archaeonantica 18).

S. MArlier S. (dir.), Arles-Rhône 3, du fleuve au musée - Journal de bord d'une opération archéologique hors du commun. 1 er septembre 2004 - 4 octobre 2013, Gent, Snoek, 2018.

L. LONG, P. PICARD (dir.), César. Le Rhône pour mémoire. Vingt ans de fouilles dans le fleuve à Arles, catalogue d'exposition, Arles, Musée départemental Arles Antique, Actes Sud, 2009.

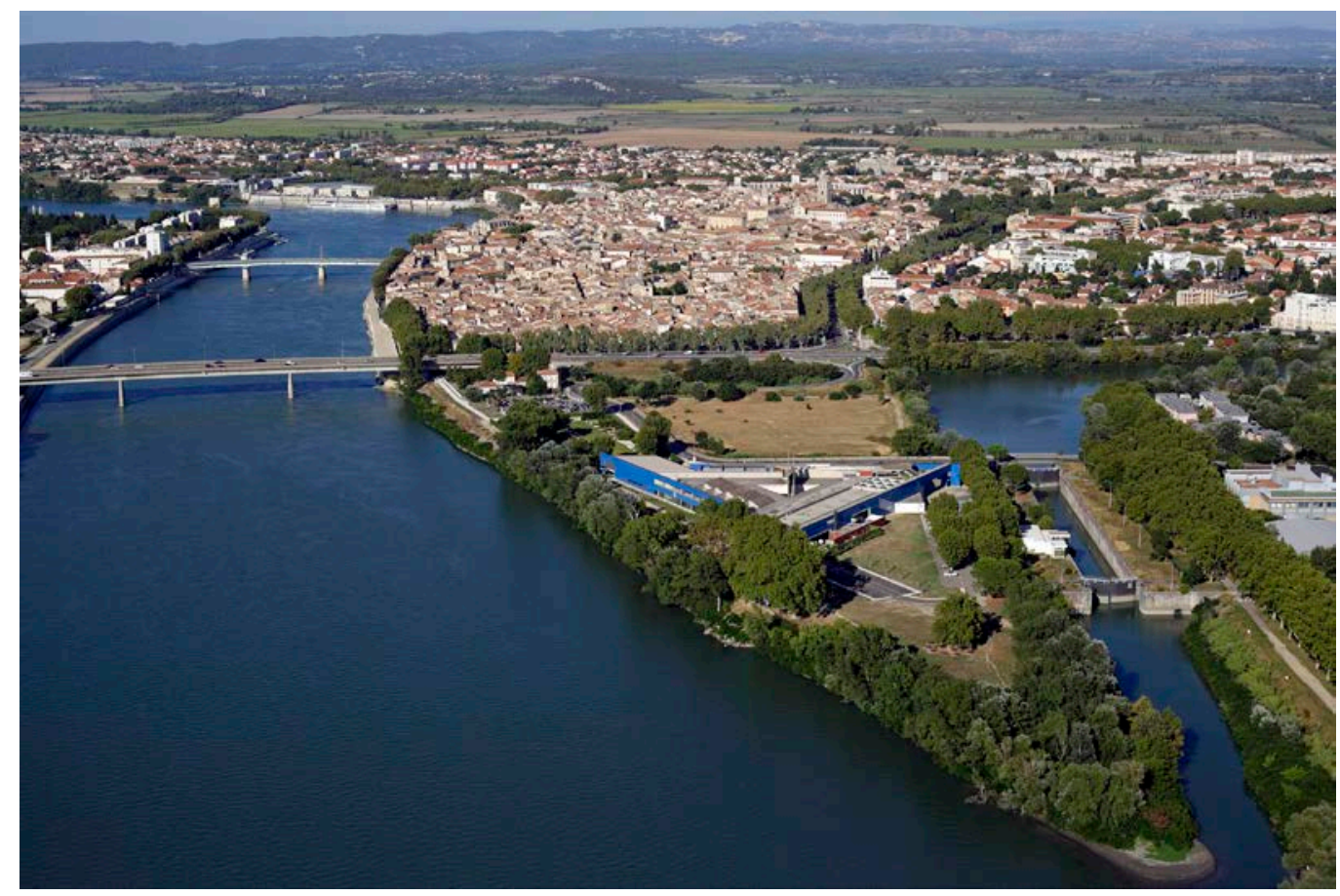

Vue, au premier plan, du musée départemental Arles antique situé sur la rive gauche du Rhône et la ville d'Arles en arrière plan (cliché R. BÉNALI, Mdaa / CD13).

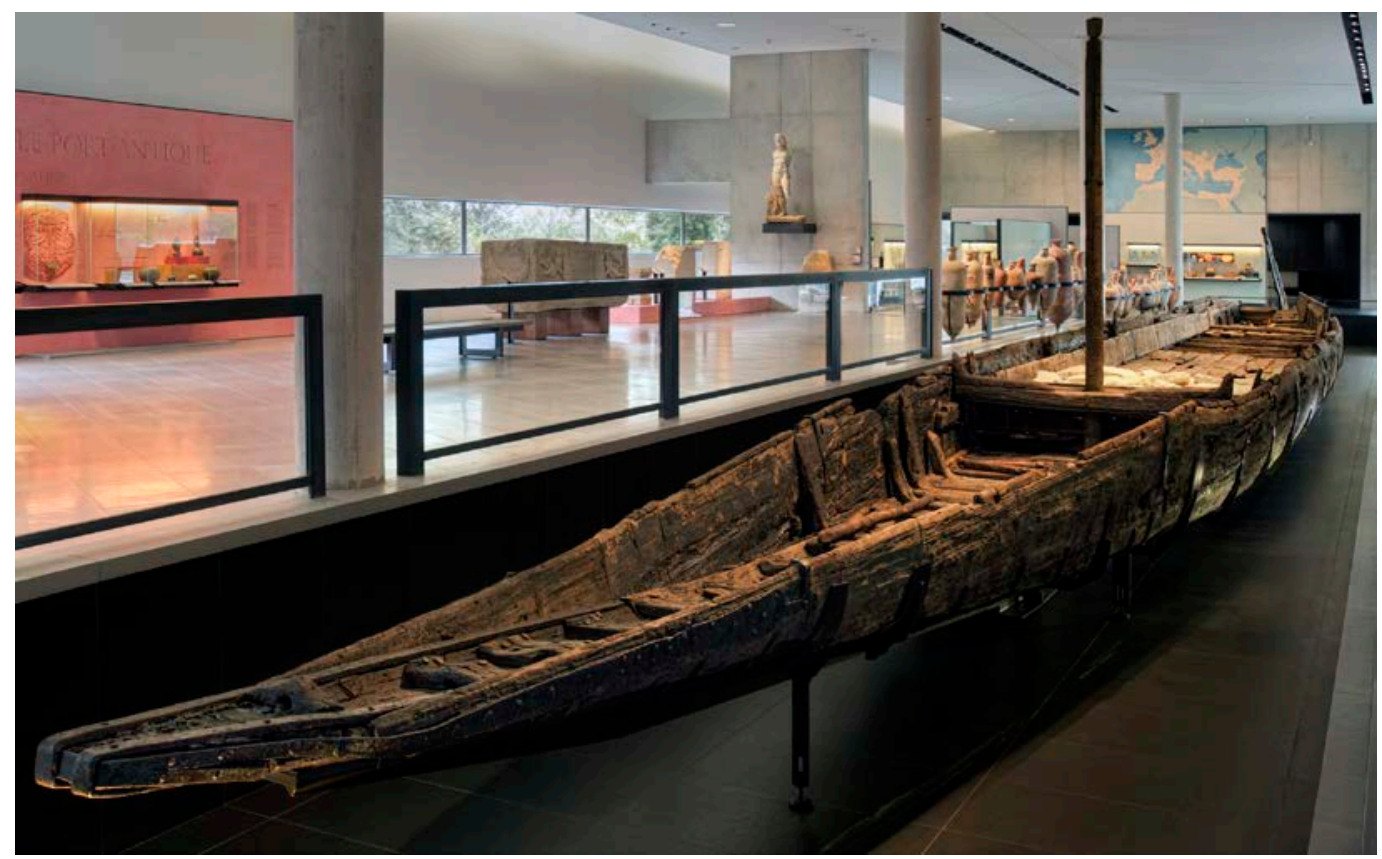

Le chaland gallo-romain Arles-Rhône 3 dans la nouvelle aile du musée dédiée au port fluviomaritime d'Arles de l'époque romaine (cliché R. BÉNALI, Mdaa / CD13). 


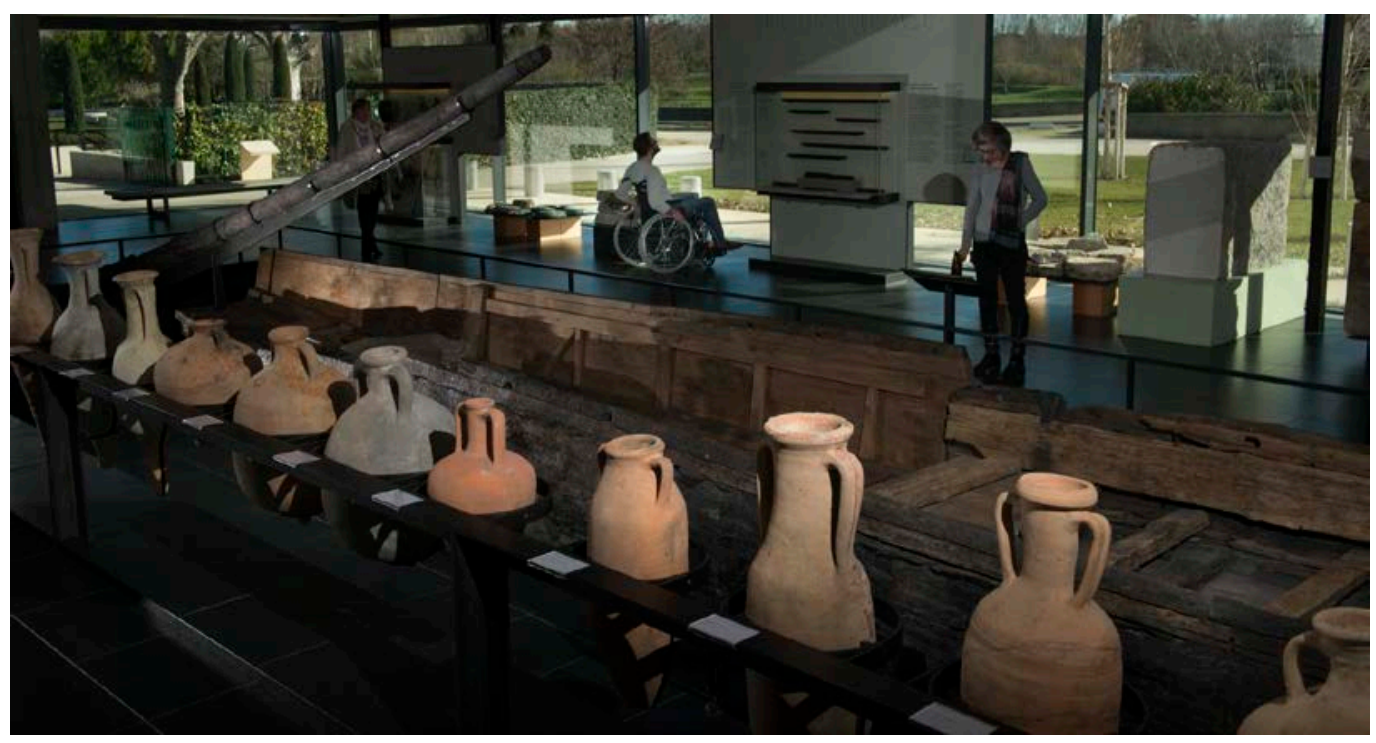

Cliché L. Roux, Mdaa / CD13.

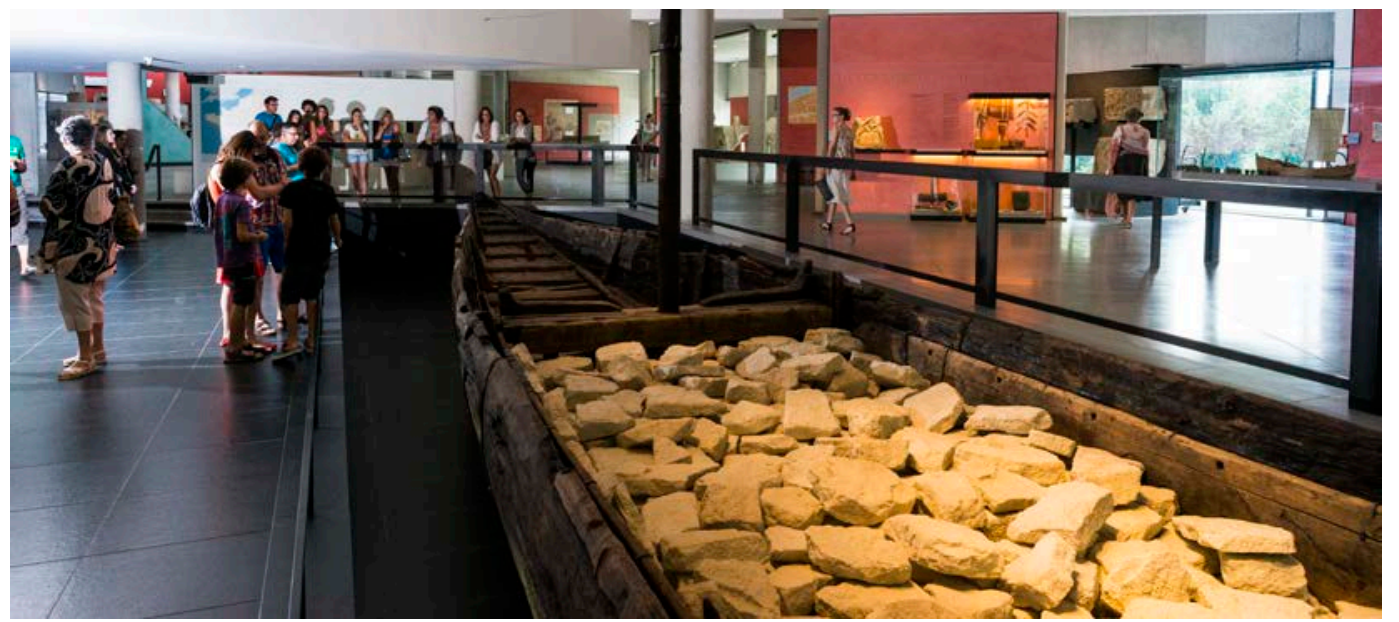

Cliché L. Roux, Mdaa / CD13. 